\title{
Acupuncture and pain (survey on literature)
}

\section{Introduction}

Physical/emotional pain used to be a common complain for many people all over the world- More different national surveys found that more than $25 \%$ of adults had recently experienced some pain lasting longer time as one day. The conventional treatments are the follows: such as prescription pharmacological substances medications, but people are to go to try different complementary treatments. One of them is as acupuncture in an effort to relieve pain. This fact sheet provides more basic information about relationship of pain and applied acupuncture. Now, we are going to summarize scientific research on acupuncture for specific kinds of pain, and give some sources for additional information.

To summarize the complexity of pain- developing any kind of pain syndrome-they are several important factors: (Figure 1).

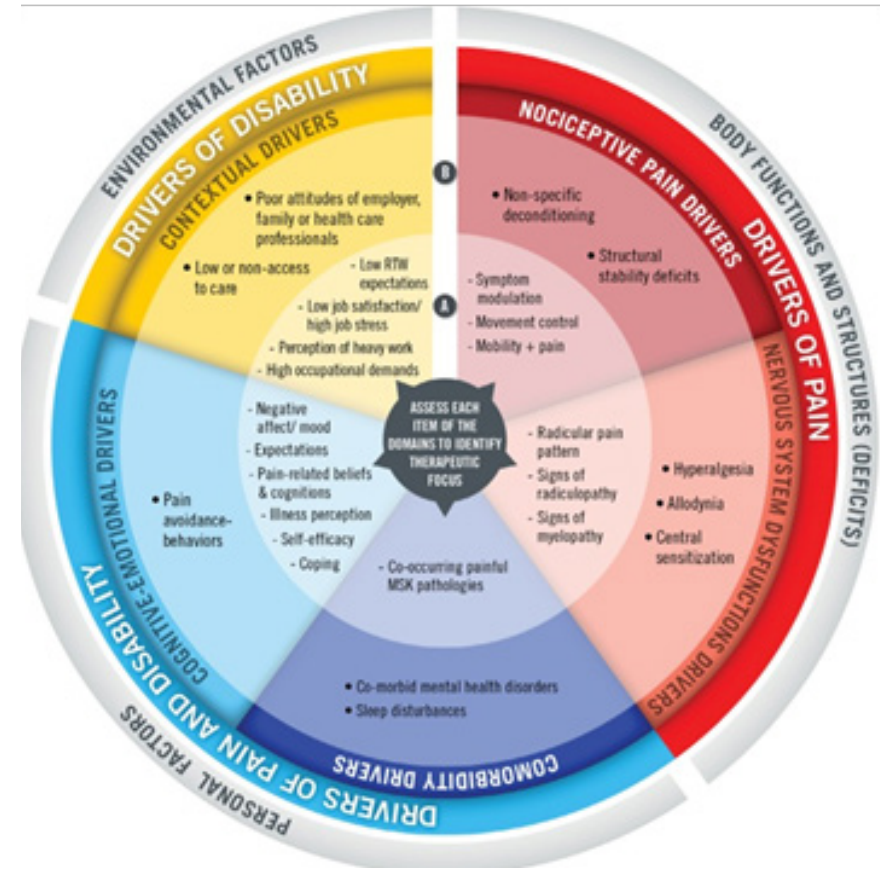

Figure I Key Points for recent study are the follows: acupuncture and pain.

People use acupuncture for various types of pain. Back pain is one of the most commonly reported uses, followed by others as knee, hip joint-, neck pain-, and headache. Of course, acupuncture used for other type of diseases as insomnia, neurological disturbances, gastroenterological problems, but they are not the task of recent summary.

Acupuncture is being studied for its efficacy in alleviating many kinds of pain. There are promising findings in some conditions according to scientific publication in this field. We can find positive results in chronic low-back pain and osteoarthritis of the knee; but for most other conditions, but additional researches are needed. The former called "National Center for Complementary and Alternative Medicine" (NCCAM) (now its newer name is: National Center for Integrated Medicine) sponsored a wide range of different levels on acupuncture research. Acupuncture is generally considered to be
Volume II Issue 4 - 2018

\author{
Gabriella Hegyi, Szőke Henrik, Kovács Zoltán \\ Pecs TCM Confucius Institute, Pecs University, Hungary, \\ Hungary,
}

Correspondence: Hegyi Gabriella, Pecs TCM Confucius Institute, Pecs University, Hungary, Email drhegyi@hu.inter.net

Received: June 29, 2018 | Published: July 19, 2018

safe when it is performed correctly. (Depends from skillfulness of practitioners and schools).

According to the Traditional Chinese Medicine theory (which is more than over 5000years old), acupuncture regulates "the flow of "Qi"-(which means the vital energy) in the organism. Researches to test scientific theories about how acupuncture might work to relieve pain are permanently growing.

\section{What does it mean: pain?}

Pain is a feeling, which is facilitated in the nervous system via sensor pathway. It may be sharp or dull, off-and-on or steady, localized on any part of the organism (such as back pain) or all over (such as muscle pains from the flu). Sometimes, pain alerts patients to injuries and illnesses that need immediately attention. Although, pain usually goes away once the underlying problem is addressed. Pain can last for days, weeks, months, or even for many years. The chronic pain may be due to an ongoing condition (such as arthritis, chronic degenerative musculoskeletal disturbances) or to abnormal activity in pain-sensing regions of the brain, or sometimes, he cause may not be known (psychosomatic origin).

For relieving their pain, many patient take over-the-counter medications-either acetaminophen or non-steroidal anti-inflammatory drugs (NSAIDs, including aspirin, naproxen, and ibuprofen). Stronger medications, including NSAIDs in higher dosages and narcotics, are available by prescription only, but we should take into account the side effects (heavy or silent gastrointestinal problems) People may also try non-drug approaches for helping to relieve their pain. Other solutions are the follows: including physical and occupational therapy, cognitive behavioral therapy, self-care techniques, and CAM therapies such as spinal manipulation (Tuina,- which is the part of the Traditional Chinese Medicine- Manual Medicine, Osteopathy )or different types of acupuncture (dry-needle, laser, permanent monofilament-needle, acu-injections, neuraltherapy with small dosage of Procain, Lidocain, Xyloneural (1\%), et.

\section{Usage of acupuncture for pain}

The dry needling, as the Acupuncture, situated among the oldest healing practices in the world, (officially a part of the World Heritage) is a part of so called "Traditional Chinese Medicine" which is a complex system. Acupuncture practitioners stimulate specific areas on the body-most often they insert thin needles through the skin- deep is different- to the so called "acupuncture points". According to the 
"Traditional Chinese medicine theory", this regulates the flow of qi (which means the vital energy of the body) along pathways known as "Meridians", which are basically the sequences of the acupuncture points centripetally/centrifugally.

According to the CAMrella Pan-European Survey, which included a comprehensive survey of CAM use by 29countries, (2010-2012) 2,8 percent of respondents (an estimated 6,2million Europeans) said they had ever used acupuncture in the past years. A special analysis of acupuncture data found that pain or musculoskeletal complaints accounted for 7 of the top 10 conditions for which people turn to acupuncture treatments. Back pain was the most common occurring disease; followed by joint-, neck pain, severe headache/migraine attacks, and recurring pain. (According to D. Eisenberg's Study, Harvard Uni).

\section{What the science says about acupuncture for pain?}

This discussion has a long story and has been continuing or a longer time between scientist and skepticisms. Acupuncture has been studied for a wide range in different not only of pain conditions, such as postoperative dental pain, fibromyalgia, carpal tunnel syndrome, headache, low-back pain, menstrual spastic complains, myofascial pain, chronic degenerative diseases of articulations, discopathy, osteoarthritis, and tennis elbow.

Overall, it can be very difficult to compare different levels of acupuncture research results from study to study and to draw conclusions from the cumulation of evidence. This is because studies may use different acupuncture techniques (e.g., electrical vs. manual, permanent, acu-injections, soft laser beam, acupressure massage techniques), controls (with comparison groups, sham groups), and outcome measures. One particularly complex factor in acupuncture research is choosing the controls - as elevate quality of trial. The choice depends in part on whether the authors want to research a particular aspect of applied acupuncture (e.g., special brain effects) or to determine whether acupuncture is useful compared with other health care forms. For example the control group has not received acupuncture treatment, which arises ethical problems. Examples of control groups include study participants who receive no acupuncture, simulated acupuncture (procedures that sham acupuncture, sometimes also referred to as "placebo" or "sham"). The main question remains that acupuncture according to special "pattern" in TCM is an "individual treatment" and no 2 patients with the same complains but different conditions can receive the same points combination.! Very promising method is the measure the pulse waves before and after acupuncture treatment to estimate the positive effect of intervention.

Acupuncture researches have an emerging theme, which is the role of the placebo. For example, a 2009 systematic review of research on the pain-relieving effects of acupuncture compared with placebo (simulated) or no acupuncture was inconclusive. The authors of reviews found only a small difference between acupuncture and placebo. Also, they found a moderate difference between placebo and "no acupuncture" treatments. The effect of placebo acupuncture varied considerably, and the effect of acupuncture appeared unrelated to the specific kind of used placebo procedure. All of the study participants received standard care. Typically were consisting of different analgesic drugs and physical therapy. This study has a misinterpretation of fact that any kind of needling (even thought sham form) can be a part of the various so-called microsystem of acupuncture, which is not understood properly! In general, acupuncture appears to be a promising alternative/complementary method for some of these pain conditions; however, further research is needed.

\section{About scientific evidence on cam therapies}

Scientific evidence on CAM therapies includes results from experimental research in laboratory as well as studies, case stories, double blind clinical trials, systematic reviews (published in any professional journals). It encompasses both "positive" findings (evidence that a therapy may work) and "negative" findings (evidence that it probably does not work or that it may be unsafe).

Scientific journals publish only proved study results, as well as review articles that evaluate the evidence as it accumulates; information about CAM research primarily on the most rigorous review articles, known as systematic reviews and meta-analyses, which number is growing permanently all over the world, recently this number is over 3 million, in acupuncture literature more than 23000 .

\section{The diseases where the acupuncture treatment advised by WHO (World Health Organization)according literature survey}

\section{Carpal tunnel syndrome}

Although an older 1997 consensus statement on acupuncture concluded that acupuncture was promising for carpal tunnel syndrome, additional research confirming acupuncture's efficacy for this condition is scant. In addiction, Neuraltherapy (Procain or Lidocain in $1 \%$ solution) can be added for better results are combined with acupuncture.

\section{Fibromyalgia}

Evidence on acupuncture treatment applying for fibromyalgic cases is doubt was in past. Some reviews in the scientific literature have found the evidence is getting to be very promising. However, another review that focused on the few rigorous randomized controlled trials on acupuncture, as an adjunct therapy for fibromyalgia did not find enough benefit for it. Additionally, a 2003 assessment by the Agency for Healthcare Research and Quality concluded that the those evidence was insufficient. The beneficial effects of acupuncture according to fibromyalgia can not be determined. Nowadays, it seems to be accepted treatment again the application of acupuncture! Lately, in new German study the acupuncture is effective for this disease.

\section{Low-back pain}

According to clinical practice guidelines issued by the American Pain Society and the American College of Physicians in 2007, acupuncture is one of several CAM therapies. Physicians should be considered when patients with chronic low-back pain do not respond to conventional management-to apply this treatment In early, few studies, combining actual acupuncture with conventional treatment was more effective than conventional treatment alone for relieving chronic low-back pain; but actual acupuncture was not more effective than sham/simulated acupuncture or conventional treatment. However, a large, rigorously designed clinical trial reported in May 2009 found that actual acupuncture and simulated acupuncture were equally effective-and both were more effective than conventional treatment- 
for relieving chronic low-back pain. There is insufficient evidence to draw definite conclusions about the effectiveness of acupuncture for acute low-back pain, but in chronic pain condition it is useful and advisable method to alleviate pain.

\section{Headache/migraine}

Study results on acupuncture for headache are conflicting. Some literature reviews found evidence to support the use of acupuncture for headache, but others noted that most of the studies were of poor quality. A 2008 review of randomized trials on acupuncture highlighted a few well-designed trials whose findings indicate that acupuncture reduces migraine symptoms and is as effective as headache medications. In addition, a 2009 review found that acupuncture helps relieve tension headaches, where it is a promising method to alleviate symptoms. However, two large trials that looked at acupuncture for migraines found no difference between actual and sham acupuncture, both of which were equal to conventional care or superior to no treatment. In tension headache the acupuncture can diminish and alleviate the attacks significantly.

\section{Menstrual cramps}

Two literature reviews have suggested that acupuncture may help with pain from menstrual cramps, but the research is limited, lately they are a few better trial with higher significance. Newer founding are that acupuncture can influence hormone levels which pay significant role in this condition.

\section{Myofascial pain}

The evidence for acupuncture and myofascial pain (in which pain occurs in sensitive areas, known as special trigger points in any part of body, in the muscles) is mixed. Some literature reviews have found the evidence promising, but another review indicated that "needling therapies" for myofascial trigger point pain were not more effective than placebo. Newer trials show improving symptoms after acupuncture from Germany.

\section{Neck pain}

Studies of acupuncture for chronic neck pain have found that acupuncture provided better pain relief than some simulated treatments. However, the studies varied in terms of design and most had small sample sizes. The combination of acupuncture with manual therapy/Neuraltherapy seems to be more effective.

\section{Osteoarthritis/chronic knee pain}

Acupuncture appears to be effective for osteoarthritis, particularly in the area of knee pain. Recent literature reviews have found that acupuncture provides pain relief. Additionally, it can improve function for patients suffering from osteoarthritis in the knee. However, authors of a 2014 systematic literature review suggested that although some large, high-quality trials have shown that acupuncture may be effective for osteoarthritis of the knee. They are more differences in the design, size, and protocol of the studies make it hard to draw any definite conclusions from the body of research. These authors made a conclusion that it was soon to recommend the application of acupuncture as a routine part of care for patients with osteoarthritis, but it is advisable to postpone operation in younger age!

\section{Postoperative dental pain:}

Recent data on acupuncture for postoperative dental pain are scant. Although literature reviews based on earlier evidence have identified acupuncture as a promising treatment for dental pain. Data said especially pain following and before tooth extraction. For example, a 1999 study of 39 dental surgery patients found that acupuncture was superior to placebo (simulated acupuncture) in prevention of postoperative pain. However, a 2005 study of 200 dental surgery patients found no significant analgesic effect for acupuncture compared to simulated acupuncture, although patients who believed they received acupuncture reported significantly less pain than those who believed they received a placebo. In case of allergy to local anesthetics acupuncture can be a useful solution for dental practice.

\section{Tennis elbow}

Study results on the use of acupuncture for tennis elbow (lateral epicondyle) pain are mixed, but some of them is real promising. An early review of clinical trials reported that data on acupuncture for lateral epicondyle pain were insufficient and of poor quality; however, recent reviews have found the evidence promising, noting strong evidence that acupuncture provides short-term pain relief for lateral epicondyle pain. Other authors reported positive effect of acupuncture and Neuraltherapy/ soft laser usage in combination in theses cases. Acupuncture has also been studied for a variety of other pain conditions, including arm and shoulder pain, (Capsulitis chronica) pregnancy-related pelvic and back pain, and temporo-mandibular joint (jaw) dysfunction. Although earlier some studies have produced some positive results, more evidence was given later to determine the efficacy of acupuncture for any of these conditions.

There is evidence that patient's attitudes and positive imagination about acupuncture can affect positively the all of outcome. In a 2015 study, researchers analyzed data from four clinical trials of acupuncture for various types of chronic pain. Participants had been asked whether they expected acupuncture to help their pain. In all four trials, those with positive expectations reported significantly greater pain relief. It is seems to be to so called "placebo effect", which should not been underestimated in any intervention. Placebo has a big role in any form of treatment. Basically, we can tell that acupuncture can affect quality of life of patients with influencing autonomic nervous system, higher release of neurotransmitters, hormone and endomorphic release.

Furthermore, studying the acupuncture's efficacy, researchers are looking at potential bio mechanisms-that is, how acupuncture might work to diminish and relieve any kind of pain. There are several theories about these bio mechanisms (e.g., acupuncture activates opioid systems in the brain that respond to pain, like Control Gate theory (Melczack and Wall), augmentation effect); additional research are available by now to punctuate this theories. Researchers lately are using the modern neuroimaging techniques such as functional magnetic resonance imaging (fMRI) to investigate the effects of Acupuncture on various regions and part of the brain. In 2015, the "Neurobiological Correlates of Acupuncture" conference was organized to discuss research challenges and directions in acupuncture neuroimaging researches. References to above mentioned: it is really very interesting that a point below ancle (Urine Bladder 60) corresponds to activation areas in nucleus thalamus!

\section{Side effects and risks}

Acupuncture is generally considered to be safe for patients when performed by an experienced, high skilled practitioner. Should be used any time sterile needles and appropriate techniques. Relatively it was only a few complications from acupuncture they were reported by some authors Serious adverse events related to acupuncture are 
relative rare, but include sometimes purulent infections (non sterile needles!) and punctured organs. (like pneumothorax, which needs immediately hospitalization)

Additionally, there are fewer adverse effects associated with acupuncture than with many drug treatments (such as antiinflammatory medication and steroid tablets or injections) used to manage painful musculoskeletal conditions like fibromyalgia, myofascial pain, low back pain, osteoarthritis, and tennis elbow.

The mortality due to side effects of pharmacological intervention is relative higher as acupuncture.

Lately, several studies using fMRI technology to study brain activity during acupuncture, including in people with pain conditions such as fibromyalgia and osteoarthritis which is promising development to understand "what we do and how?" with acupuncture.

The old experiments today need modernization, scientific explanation on side of Evidence Based Medicine to be more accepted and used parallel for benefit of the patients.

\section{Acknowledgements}

None.

\section{Conflict of interest}

The author declares no conflict of interest.

\section{References}

1. Barnes PM, Bloom B, Nahin R. Complementary and alternative medicine use among adults and children: United States, 2007. National Health Statistics Report. 2008;12:1-23.

2. Birch S, Hesselink JK, Jonkman FA, et al. Clinical research on acupuncture. Part 1. What have reviews of the efficacy and safety of acupuncture told us so far? Journal of Alternative and Complementary Medicine. 2004;10(3):468-480.

3. Burke A, Upchurch DM, Dye C, et al. Acupuncture use in the United States: findings from the National Health Interview Survey. Journal of Alternative and Complementary Medicine. 2006;12(7):639-648.

4. Ernst E. Acupuncture-a critical analysis. Journal of Internal Medicine. 2006;259(2):125-137.

5. Lao L, Hamilton GR, Fu J, et al. Is acupuncture safe? A systematic review of case reports. Alternative Therapies in Health and Medicine. 2003;9(1):72-83.

6. Linde K, Witt CM, Streng A, et al. The impact of patient expectations on outcomes in four randomized controlled trials of acupuncture in patients with chronic pain. Pain. 2007;128(3):264-271

7. Madsen MV, Gøtzsche PC, Hróbjartsson A. Acupuncture treatment for pain: systematic review of randomized clinical trials with acupuncture, placebo acupuncture, and no acupuncture groups. BMJ. 2009; 338:a3115.

8. MacPherson H, Nahin R, Paterson C, et al. Developments in acupuncture research: big-picture perspectives from the leading edge. Journal of Alternative and Complementary Medicine. 2008:14(7):883-887.

9. Napadow V, Ahn A, Longhurst J, et al. The status and future of acupuncture mechanism research. Journal of Alternative and Complementary Medicine. 2008:14(7):861-869.

10. Napadow V, Webb JM, Pearson N, et al. Neurobiological correlates of acupuncture: November 17-18, 2005. Journal of Alternative and Complementary Medicine. 2006;12(9): 931-935.
11. National Center for Health Statistics. Health, United States, 2006, With Chartbook on Trends in the Health of Americans. Special feature: pain Hyattsville: National Center or Health Statistics; 2006. p. 68-87.

12. National Institutes of Health Consensus Panel. Acupuncture: NIH Consensus Development Conference Statement. Nov. 3-5, 1997. 15(5):134.

13. Park J, Linde K, Manheimer E, et al. The status and future of acupuncture clinical research. Journal of Alternative and Complementary Medicine. 2008;14(7):871-881

14. Muller M, Tsui D, Schnurr R, et al. Effectiveness of hand therapy interventions in primary management of carpal tunnel syndrome: a systematic review. Journal of Hand Therapy. 2004;17(2):210-228.

15. Agency for Healthcare Research and Quality. Technology Assessment: Acupuncture for Fibromyalgia. Rockville: Agency for Healthcare Research and Quality; 2003.

16. Assefi NP, Sherman KJ, Jacobsen C, et al. A randomized clinical trial of acupuncture compared with sham acupuncture in fibromyalgia. Annals of Internal Medicine. 2005;143(1):10-19.

17. Harris RE, Gracely RH, McLean SA, et al. Comparison of clinical and evoked pain measures in fibromyalgia. The Journal of Pain. 2006;7(7):521-527.

18. Harris RE, Tian X, Williams DA, et al. Treatment of fibromyalgia with formula acupuncture: investigation of needle placement, needle stimulation, and treatment frequency. Journal of Alternative and Complementary Medicine. 2005;11(4):663-671.

19. Mayhew E, Ernst E. Acupuncture for fibromyalgia-a systematic review of randomized clinical trials. Rheumatology (Oxford). 2007;46(5):801-804.

20. Headache/Migraine.

21. Coeytaux RR, Kaufman JS, Kaptchuk TJ, et al. A randomized, controlled trial of acupuncture for chronic daily headache. Headache. 2005;45(9):1113-1123.

22. Diener HC, Kronfeld K, Boewing G, et al. Efficacy of acupuncture for the prophylaxis of migraine: a multicentre randomized controlled clinical trial. Lancet Neurology. 2006;5(4):310-316.

23. Endres HG, Diener HC, Molsberger A. Role of acupuncture in the treatment of migraine. Expert Review of Neurotherapeutics. 2007;7(9):1121-1134.

24. Griggs C, Jensen J. Effectiveness of acupuncture for migraine: critical literature review. Journal of Advanced Nursing. 2006;54(4):491-501.

25. Linde K, Allais G, Brinkhaus B, et al. Acupuncture for tension-type headache. Cochrane Database of Systematic Reviews. 2009;(1):CD007587.

26. Linde K, Streng A, Jürgens S, et al. Acupuncture for patients with migraine: a randomized controlled trial. Journal of the American Medical Association. 2005;293(17):2118-2125.

27. Melchart D, Streng A, Hoppe A, et al. Acupuncture in patients with tensiontype headache: randomized controlled trial. BMJ. 2005;331(7513):376382.

28. Chou R, Qaseem A, Snow V, et al. Diagnosis and treatment of low back pain: a joint clinical practice guideline from the American College of Physicians and the American Pain Society. Annals of Internal Medicine. 2007;147(7):478-491.

29. Eisenberg DM, Post DE, Davis RB, et al. Addition of choice of complementary therapies to usual care for acute low back pain: a randomized controlled trial. Spine. 2007;32(2):151-158.

30. Furlan AD, van Tulder M, Cherkin D, et al. Acupuncture and dry-needling for low back pain: an updated systematic review within the framework of the Cochrane collaboration. Spine. 2005;30(8):944-963. 
31. Manheimer E, White A, Berman B, et al. Meta-analysis: acupuncture for low back pain. Annals of Internal Medicine. 2005;142(8):651-663.

32. Proctor ML, Smith CA, Farquhar CM, et al. Transcutaneous electrical nerve stimulation and acupuncture for primary dysmenorrhoea. Cochrane Database of Systematic Reviews. 2002;(1):CD002123.

33. White AR. A review of controlled trials of acupuncture for women's reproductive health care. The Journal of Family Planning and Reproductive Health Care. 2003;29(4):233-236.

34. Witt CM, Reinhold T, Brinkhaus B, et al. Acupuncture in patients with dysmenorrhea: a randomized study on clinical effectiveness and cost-effectiveness in usual care. American Journal of Obstetrics and Gynecology. 2008;198(2):166.e1-166.e8.

35. Cummings TM, White AR. Needling therapies in the management of myofascial trigger point pain: a systematic review. Archives of Physical Medicine and Rehabilitation. 2001;82(7):986-992.

36. Trinh KV, Graham N, Gross AR, et al. Cervical Overview Group. Acupuncture for neck disorders. Cochrane Database of Systematic Reviews. 2006;3:CD004870.

37. Osteoarthritis/Knee Pain.

38. Agency for Healthcare Research and Quality. Technology Assessment: Acupuncture for Osteoarthritis. Rockville: Agency for Healthcare Research and Quality; 2003.

39. Berman BM, Lao L, Langenberg P, et al. Effectiveness of acupuncture as adjunctive therapy in osteoarthritis of the knee: a randomized, controlled trial. Annals of Internal Medicine. 2004;141(12):901-910.

40. Bjordal JM, Johnson MI, Lopes-Martins RA, et al. Short-term efficacy of physical interventions in osteoarthritic knee pain. A systematic review and meta-analysis of randomised placebo-controlled trials. $B M C$ Musculoskeletal Disorders. 2007;8:51.

41. Foster NE, Thomas E, Barlas P, et al. Acupuncture as an adjunct to exercise based physiotherapy for osteoarthritis of the knee: randomised controlled trial. BMJ. 2007;335:436.

42. Kwon YD, Pittler MH, Ernst E. Acupuncture for peripheral joint osteoarthritis: a systematic review and meta-analysis. Rheumatology (Oxford). 2006;45(11):1331-1337.

43. Manheimer E, Linde K, Lao L, et al. Meta-analysis: acupuncture for osteoarthritis of the knee. Annals of Internal Medicine . 2007;146(12):868877.
44. Scharf HP, Mansmann U, Streitberger K, et al. Acupuncture and knee osteoarthritis: a three- armed randomized trial. Annuals of Internal Medicine. 2006;145(1):12-20.

45. White A, Foster NE, Cummings M, et al. Acupuncture treatment for chronic knee pain: a systematic review. Rheumatology (Oxford). 2007;46(3):384-390.

46. Bausell RB, Lao L, Bergman S, et al. Is acupuncture analgesia an expectancy effect? Preliminary evidence based on participants' perceived assignments in two placebo- controlled trials. Evaluation and the Health Professions. 2005:28(1):9-26.

47. Lao L, Bergman S, Hamilton GR, et al. Evaluation of acupuncture for pain control after oral surgery: a placebo-controlled trial. Archives of Otolaryngology Head \& Neck Surgery. 1999;125(5):567-572.

48. Rosted P. The use of acupuncture in dentistry: a review of the scientific validity of published papers. Oral Diseases. 1998;4(2):100-104.

49. Bisset L, Paungmali A, Vicenzino B, et al. A systematic review and meta-analysis of clinical trials on physical interventions for lateral epicondylalgia. British Journal of Sports Medicine. 2005;39(7):411-422.

50. Green S, Buchbinder R, Barnsley L, et al. Acupuncture for lateral elbow pain. Cochrane Database of Systematic Reviews. 2002;(1):CD003527.

51. Trinh KV, Phillips SD, Ho E, et al. Acupuncture for the alleviation of lateral epicondyle pain: a systematic review. Rheumatology (Oxford). 2004;43(9):1085-1090.

52. Trudel D, Duley J, Zastrow I, et al. Rehabilitation for patients with lateral epicondylitis: a Systematic review. Journal of Hand Therapy. 2004;17(2):243-266.

53. Ee CC, Manheimer E, Pirotta MV, et al. Acupuncture for pelvic and back pain in pregnancy: a systematic review. American Journal of Obstetrics and Gynecology. 2008;198(3):254-259.

54. Ernst E, White AR. Acupuncture as a treatment for temporomandibular joint dysfunction: a systematic review of randomized trials. Archives of Otolaryngology Head \& Neck Surgery. 1999;125(3):269-272.

55. Goldman RH, Stason WB, Park SK, et al. Acupuncture for treatment of persistent arm pain due to repetitive use: a randomized controlled clinical trial. The Clinical Journal of Pain. 2008;24(3):211-218.

56. Green S, Buchbinder R, Hetrick S. Acupuncture for shoulder pain. Cochrane Database of Systematic Reviews. 2005;2:CD005319. 